\title{
Development and clinical evaluation of bipolar radiofrequency cryptolysis
}

\author{
Klaus Vogt ${ }^{* 1}$, Svetlana Konuhova ${ }^{1}$, Kaspars Peksis ${ }^{1}$ and J. Markow ${ }^{2}$ \\ ${ }^{1}$ Center of Experimental Surgery, University of Latvia, Kalnciema iela 98, Riga 1014, Latvia \\ ${ }^{2}$ Anatomy and Histology Department, Faculty of Medicine, University of Latvia, Riga, Latvia
}

\begin{abstract}
Objectives: Cryptolysis is a highly efficient conservative procedure in halitosis caused by deep tonsillar crypts or tonsilloliths. It can be carried out by laser, coblation or radiofrequency application, with radiofrequency application being the most cost effective. Monopolar methods generate a wider electrical field with more side effects; therefore, it is necessary to develop and test a new bipolar electrode.
\end{abstract}

Methods: In a prospective non-randomized study seventy-one patients were treated with a monopolar probe-like electrode and three radiofrequency generators. Sixteen patients underwent treatment with a bipolar electrode. In total, 200--300 J was applied. Tonsil specimens underwent histology.

Results: Application of a bipolar electrode led to better results than those elicited with a monopolar electrode for cryptolysis.

Conclusion: Bipolar radiofrequency cryptolysis should be the first-choice procedure for the treatment of halitosis and tonsilloliths.

\section{Introduction}

Bad breath ("halitosis", "oral malodor" or "foetor ex ore") can be a serious social problem, but is a symptom of a pathologic process only in the oral cavity $[1,2]$. Exceptions to this rule may be excessive growth of bacteria within an oropharyngeal tumor, stomatitis ulcerosa or postoperative wound-healing processes, where the cause is obvious and treatment known. Persistent halitosis can be due to suboptimal oral hygiene and impossible-to-reach areas where the nutritional base for bacteria cannot be removed (e.g., parodontopathy due to tartar and dental plaques).

Anatomic structure of the tonsils involves crypts. The latter are the ideal biotopes for growing bacteria and producing biofilms which, eventually, may be calcified and appear as "tonsil stones" or tonsilloliths. The metabolism of the content of tonsil crypts has been investigated comprehensively by Tonzetich et al. [3-9].

Halitosis and its treatment have been studied from ancient times to the contemporary practice of otorhinolaryngology [10,11]. Smears within crypts or tonsilloliths are at the borderline between normal tissue and diseased tissue. Transition to chronic tonsillitis is possible, but the prevalence of such an event is not known. Also, consensus on the definition of "chronic tonsillitis" is lacking. Crypts which must be treated are situated mostly at the upper pole of the tonsil; crypts in the middle area are cleaned by swallowing. Crypts may be much deeper than expected and can be $>1 \mathrm{~cm}$ in length (Figure 1). However, probing of crypts is not routine in investigations involving the ear, nose and throat (ENT). Several instruments have been used to remove tonsilloliths by pressing the tonsils in the sagittal direction. Tonsil-sucking tubes are part of the classic armamentarium of ENT practice (Figure 2).

\section{Cryptolysis}

The aim of the "cryptolysis" procedure is deterioration of the crypt epithelium after removal of debris or stones by preservation of adjacent tonsillar tissue and formation of a scar that closes the crypt permanently.

Several methods of cryptolysis are in use, with lasers (of different types) [12], coblation [3] and radiofrequency ablation being the most popular. Ablation of the crypt entrance has been replaced by a method involving a blunt probe-like electrode by Vogt. Both procedures are monopolar with generation of radiowaves and thermal damage to the deep layers of the tonsil (Figure 3 and 4).

In laser cryptolysis, tonsillar crypts are ablated, resulting in scarring and resolution of tonsillolith formation [3]. However, laser cryptolysis may result in crypt orifices being "welded shut". Hence, debris hidden

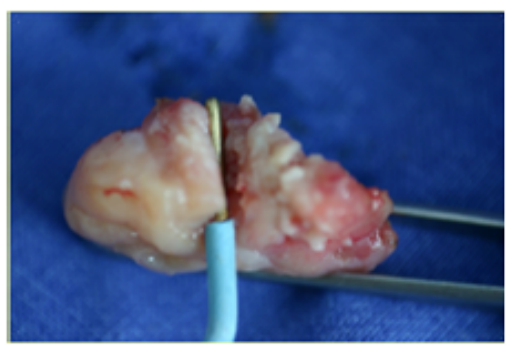

Figure 1. Crypt (depth $12 \mathrm{~mm}$ ) in a hyperplastic tonsil.

Correspondence to: Prof. Dr. Klaus Vogt, Center of Experimental Surgery, University of Latvia, Kalnciema iela 98, Riga 1014, Latvia; Tel: +49 38457 507647; Fax: +4938457 507 648; E-mail: rhinovogt@t-online.de

Key words: postural contro, rehabilitation, external focus, feed-back mechanisms

Received: November 01, 2016; Accepted: December 06, 2016; Published: December 09, 2016 


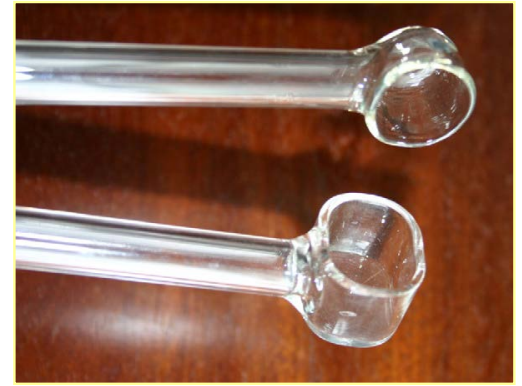

Figure 2. Glass suction devices for "tonsil sucking".

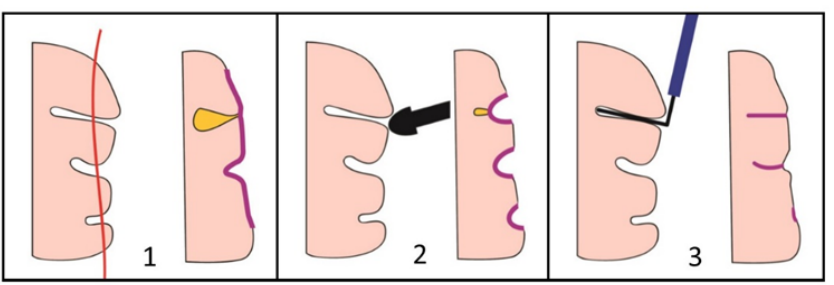

Figure 3. Different methods of cryptolysis.1, Cryptolysis with a $\mathrm{CO}_{2}$ laser.2, Circular ablation of the crypt orifice by a blunt radiofrequency electrode.3, Monopolar blunt radiofrequency electrode activated within the crypt.

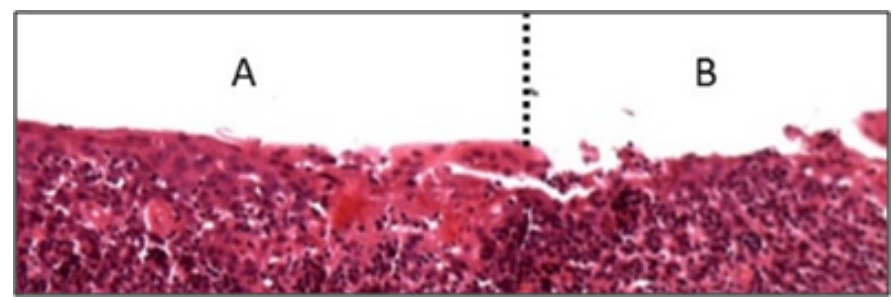

Figure 4. Radiofrequency ablation of tonsillar crypts. Destruction of the epithelium (A remains intact and $\mathrm{B}$ is destroyed).

in the crypts may be trapped, thereby creating a potential nidus for abscess formation [13]. In addition, there are potential risks: exposure to a flame in the airways; retinal damage from reflected laser scattering; oral/facial burns from unintended movement (e.g., gag reflex); management of plumes from vaporized tissues [3]. Radiofrequency ablation involves use of electrodes to obliterate crypts, removing their contents, destroying the epithelium and inducing a scarring process so that crypt openings become occluded [13]. Coblation has been described as a safe method to use. The PROcise ${ }^{\circ}$ XP Plasma Wand (Smith and Nephew, London, UK) provides the largest active surface. After dipping of the wand in saline gel, application of the wand tip to crypts results in ablation of the involved area [3].

A monopolar electrode generates a conic electrical field with an unwanted generation of steam in deeper tonsillar layers and, in some cases, induces acute postoperative tonsillitis. Therefore, a bipolar concentric electrode with a spindle-like distribution of electric waves around only the crypts has been developed by Vogt (Figure 5) [13,9].

Prototypes of the new bipolar electrode have been tested in vitro and by carrying out cryptolysis in patients immediately before a planned tonsillectomy. The histology results shown in Figures 6-9 were obtained. Primary purpose of modeling of heat transfer in the parenchyma of an organ is prediction of the level and area of potential damage caused by hyperthermia. Blood perfusion has a dominant role in enhancement of the net transfer of heat. To evaluate the heat-transfer effect of the microvascular network of the tonsils on thermal lesions, histopathologic examination of subsequently excised organs was done in 3 cases, whereas bipolar cryptolysis was followed up immediately by tonsillectomy.

Tissue samples obtained from tonsils were processed routinely followed by staining (hematoxylin and eosin) along with immunohistochemical staining for CD31 (found in endothelial cells). Distribution of the CD31-positive microvascular network in palatine tonsils was complex but followed a general theme. The parafollicular region was supplied by a dense vasculature with closely arranged pairs of arterioles and venules. Each arteriole had abundant branches and entered a follicle. These arterioles, giving off capillaries in follicles, reached the subepithelial region, where they formed a dense subepithelial capillary network. Most of the capillaries below the crypts were large and of various calibers, suggesting that these capillaries were sinusoids.

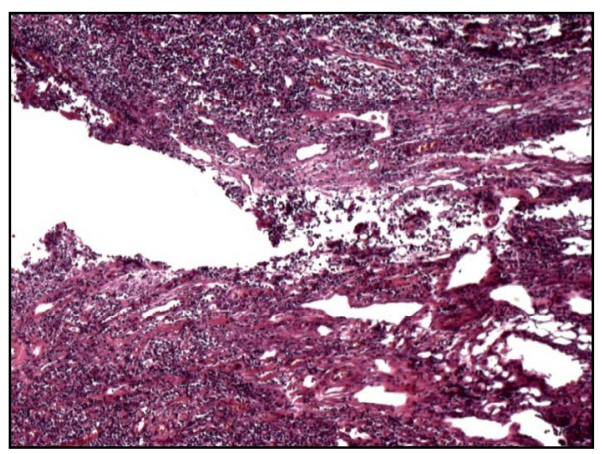

Figure 5. Coblation of tonsillar crypts. Development of steam bubbles at the electrode tip.
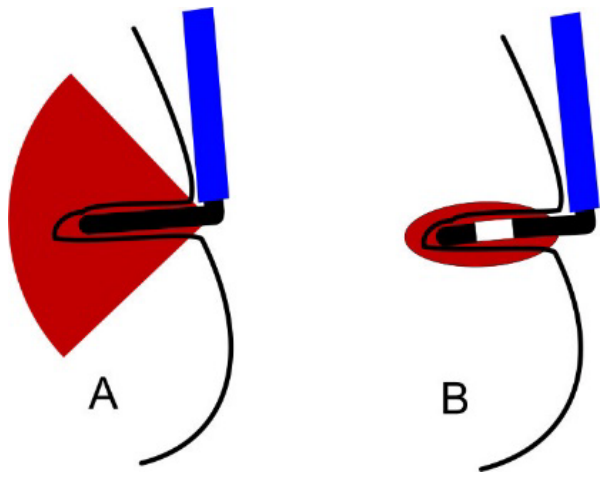

Figure 6. A probe-like monopolar electrode generates a conic electrical field with unwanted influence upon adjacent tonsillar tissue (A). A monopolar concentric electrode generates a spindle-like field around the electrode (B).

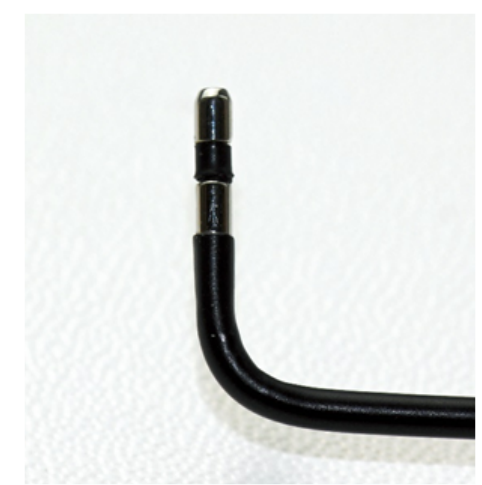

Figure 7. Active part of the bipolar cryptolysis electrode (prototype; RegerMedizintechnik, Villingendorf, Germany. 


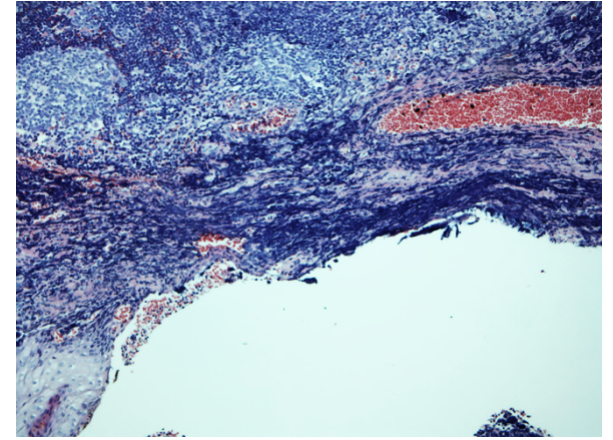

Figure 8. Elongated nuclei of a crypt-denuded area are aligned in a parallel fashion ("nuclear streaming").

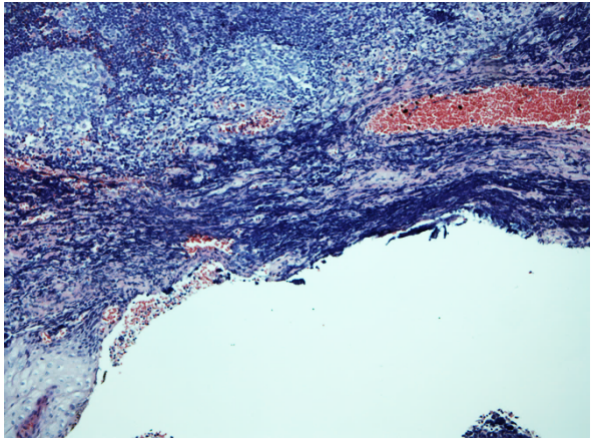

Figure 9. Zone of intramural bleeding with interfibrillar infiltration of erythrocytes Collagen fibers in the area of injury show edema.

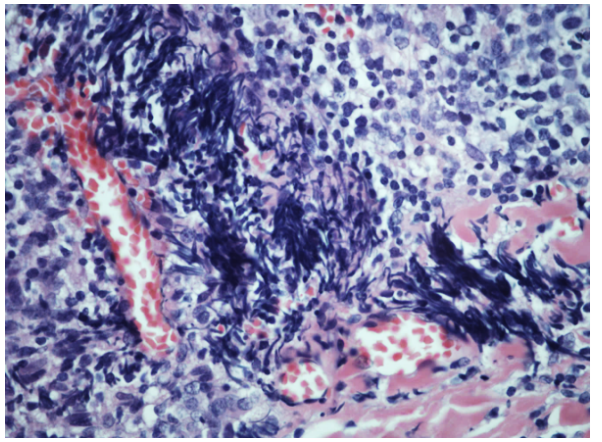

Figure 10. Internodular septa display edematous loosening of collagen fibers in the vicinity of hyperemic blood vessels. Loose cellular aggregates with "hockey stick" nuclei are seen in the area of thermoelectric injury.

In the areas of thermoelectric injury, histopathologic examination revealed edema of connective-tissue fibers in crypt-denuded area with the loss of nuclear (pyknosis, karyorrhexis, karyolysis) and cytoplasmic details of fibroblasts. Blood vessels were hyperemic and dilated, and extravasation of leukocytes and erythrocytes had occurred. In some regions of stromal connective tissue, elongated nuclei of cryptdenuded areas were aligned in a parallel fashion ("nuclear streaming"). In localized areas, internodular septa displayed edematous loosening of collagen fibers and cellular aggregates with "hockey stick" nuclei in the vicinity of hyperemic blood vessels. Due to the effects of heat, fibroblasts may have become highly susceptible to nuclear streaming from crushing by surgical instruments.

An increase in the perfusion rate can occur as a result of vasodilatation, which is a temperature-dependent thermoregulatory mechanism. Thin-walled venules could be involved directly in heat transfer into nearby tissues. Our results are in good agreement with investigations which concluded that most heat transfer occurs mainly across the walls of sinusoids and venules.

\section{Materials and methods}

In a prospective clinical study eighty-seven patients (10--79 years; 64 female, 23 male) from "Headline Clinic" (Riga, Latvia) and P. Stradinš Clinical University Hospital (Riga) formed the study cohort. Both institutions used the same methodology. The patients have been enrolled by 2 experienced surgeons responsible for the admittance of patients for tonsillectomy or tonsillotomy in a university hospital. One of those surgeons performed the surgical intervention. All patients signed a written consent for the intervention. Between April 2012 and September 2014 seventy-one patients were treated with a monopolar system, between October 2014 and February 2016 sixteen patients have been treated with a bipolar system. The monopolar electrodes have not anymore applied after starting the work with the bipolar electrode because of the good clinical results of the new method.

All patients were interviewed using a questionnaire in which questions about the period before and after manipulation and its efficacy were included.

\begin{tabular}{|l|l|}
\hline Inclusion criteria & $\begin{array}{l}\text { Visible or probable crypts with smear or stones within the upper } \\
\text { third of the tonsil, with or without minor signs of chronic or } \\
\text { chronic recurrent tonsillitis }\end{array}$ \\
\hline Exclusion criteria & $\begin{array}{l}\text { Severe chronic recurrent tonsillitis with or without abscedation. } \\
\text { Obstructive hyperplasia of the tonsils. Children under 14 years old }\end{array}$ \\
\hline
\end{tabular}

The radiofrequency generators Wavetronic 5000 (Loktal, Sao Paulo, Brasil) Surgitron RF (Ellman International, Hicksville, NY, USA) and Curis (Sutter Medizintechnik, Freiburg, Germany) were used together with the blunt monopolar cryptolysis electrode of an Ellman Intl. in an open or closed system. A total of 200--300 J of energy was applied.

After topical and local anesthesia at the upper tonsillar pole, the electrode was inserted and debris or tonsillolith removed by displacement. In some cases, tonsilloliths were removed at the beginning. The electrode was inserted and activated. The energy supply was stopped when blanching of the crypt entrance occurred. A bipolar cryptolysis electrode (Reger Medizintechnik, Villingendorf, Germany) was activated with $30 \mathrm{~W}$ for $10 \mathrm{~s}(300 \mathrm{~J})$.

\section{Results}

Figure 11 shows the distribution of preoperative complaints and the indication for cryptolysis. According to their history, $15 \%$ of patients also showed signs of chronic tonsillitis besides halitosis.

After the procedure, patients, in general, had fewer complaints then after regular tonsillectomy but sometimes developed slight pyrexia. The bipolar procedure revealed in each level of the pain-scale less days with the corresponding pain compared to the monopolar procedure. Therefore, the bipolar procedure may cause less pain than the monopolar procedure (Figure 12).

Intervention because of postoperative bleeding was not necessary.

\section{Discussion}

The desired effect of cryptolysis could be achieved in most cases, and bipolar application led to better results than monopolar application (Figure 12). Similar results have been reported recently by Krespi and Kizhner [12] as well as Chang and Thrasher [3]. If the effect is not as desired, the procedure can be repeated. Also, a dentist should 


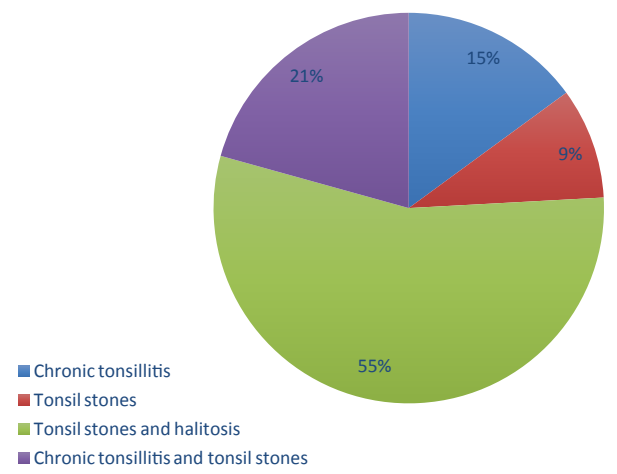

Figure 11. Patient complaints before cryptolysis.

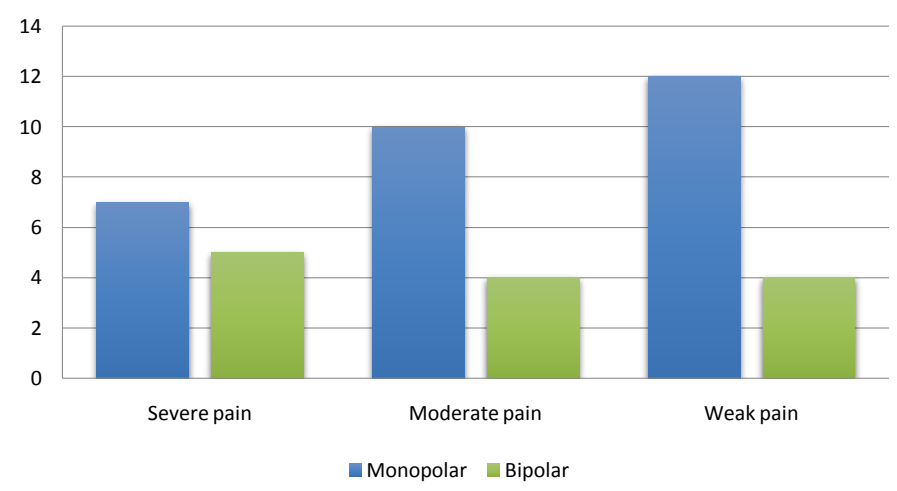

Figure 12. Painduration (days) from a three-level Likert pain-scale.
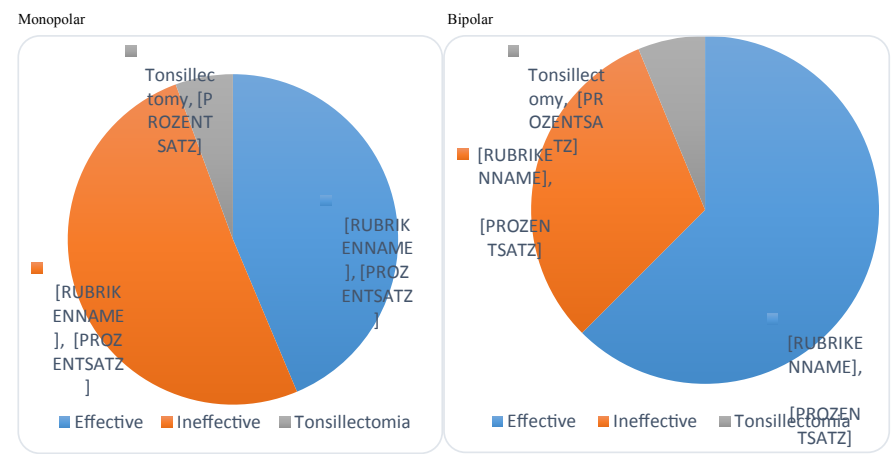

Figure 13. Efficacy of treatment using monopolar and bipolar electrodes.

ensure that plaque and interdental tartar are not the causes of halitosis. The selection of patients may improve later the statistical outcome of the cryptolysis and standardized application of energy (including an automatic cutout) may also help to improve results. However, there are as always only weak criteria for the severity of a chronic tonsillitis and the reaction towards the applied radiofrequency energy cannot be predicted. Bipolar application led to better results than those elicited using the monopolar method, what means that the intended minor lateral heat damage around the bipolar electrode parts and the hypothetic minor reaction of the tonsillar tissue could be achieved. Similar differences between monopolar and bipolar applications can be observed for the treatment of the lower turbinates, where the energy can be delivered by a bipolar double-needle electrode and the epithelium will be preserved (Vogt [13]), while monopolar applications - also known as so-called "coblation tunneling" leads to a destroyed surface similar to $\mathrm{CO}_{2}$-laser treatment with the follow-up of remarkably longer post-operative treatment. Bipolar hemostasis is nowadays the "golden standard" for the coagulation of blood vessels in sensitive areas as for instance the nose [14-17].

\section{Conclusions}

Development of a bipolar concentric electrode can improve the results of radiofrequency cryptolysis. The latter is a minimally invasive and cost-effective outpatient procedure that can reduce the number of indications for tonsillectomy.

\section{Acknowledgment}

We thank Reger Medizintechnik GmbH,Villingendorf /Germany for the cooperation during development of a new electrode.

\section{Funding sources}

The work was kindly supported by Sutter Medizintechnik GmbH, Freiburg/Germany and Reger Medizintechnik GmbH, Villingendorf/ Germany

\section{Approval by ethic committee}

The here presented study was approved by the Scientific Research Ethics Committee of University of Latvia Institute of Cardiology and Regenerative Medicine

\section{Conflict of interest}

$\mathrm{KV}$ is a consultant for S-F-X-Holding (Freiburg im Breisgau, Germany).

\section{References}

1. Porter SR, Scully C (2006) Oral malodour (halitosis). BMJ333: 632-635.[Crossref]

2. Scully C, Felix DH (2005) Oral medicine--update for the dental practitioner: oral malodour. Br Dent J 199: 498-500.[Crossref]

3. Chang CY, Thrasher R (2012) Coblation cryptolysis to treat tonsil stones: a retrospective case series. Ear Nose Throat J91: 238-254.[Crossref]

4. Loesche WJ, Kazor C (2002) Microbiology and treatment of halitosis. Periodontol 2000 28: 256-279.[Crossref]

5. Rosenberg M, McCulloch CA (1992) Measurement of oral malodor: current methods and future prospects. J Periodontol63: 776-782.[Crossref]

6. Rosenberg M, Kulkarni GV, Bosy A, McCulloch CA (1991) Reproducibility and sensitivity of oral malodor measurements with a portable sulphide monitor. $J$ Dent Res 70: 1436-1440.[Crossref]

7. Patil S, Talavera F, Kellman RM, Meyers AD, Viswanatha B (2016) Halitosis Medscape.

8. Stoodley P, Debeer D, Longwell M, Nistico L, Hall-Stoodley L, et al. (2009) Tonsillolith: not just a stone but a living biofilm. Otolaryngol Head Neck Surg 141 316-321.[Crossref]

9. Vogt K (2013) Bipolare Elektrode zur Kryptolyse der Tonsillen. German Patent 20 2013009096.0

10. Finkelstein Y, Talmi YP, Ophir D, Berger G (2004) Laser cryptolysis for the treatment of halitosis. Otolaryngol Head Neck Surg131: 372-377.[Crossref]

11. Prinz H (1930) Offensive breath, its causes and its prevention. Dent Cosmos 72:700-707.

12. Krespi YP, Kizhner V (2013) Laser tonsil cryptolysis: in-office 500 cases review. Am J Otolaryngol 34: 420-424.[Crossref]

13. Vogt K (2014) Radiofrequency surgery in otorhinolaryngology. Tuttlingen: Endopress

14. Grapp GL (1933) Fetor oris (halitosis). A medical and dental responsibility. Northwest Med J32:375-380.

15. Howe JW (1898) The breath and the diseases which give it a fetid odor. 4th ed. New York: D Appleton and Co. 
Vogt K (2016) Development and clinical evaluation of bipolar radiofrequency cryptolysis

16. Rosenberg M (1996) Clinical assessment of bad breath: current concepts. J Am Dent

Assoc 127: 475-482.[Crossref]

17. Crytolysis Doctor Directory (2016).

Copyright: $(02016$ Vogt K. This is an open-access article distributed under the terms of the Creative Commons Attribution License, which permits unrestricted use, distribution, and reproduction in any medium, provided the original author and source are credited. 